\title{
Ward Round - Late Presentation of Acute Compartment Syndrome in the Thigh \\ Jes Bates ${ }^{1}$, Biruk L Wamisho ${ }^{2}$,Meghan Griffin ${ }^{3}$,Nohakhelha Nyamulani ${ }^{1}$
}

1. University of Malawi, College of Medicine, Department of Surgery, Blantyre.

2. Addis Ababa University, Faculty of Medicine, Department of Orthopedics, Ethiopia.

3. Michigan State University, College of Osteopathic Medicine, U.S.A

Correspondence: Dr. Biruk Lambisso: lbiruklw@yahoo.com

A 36 year-old previously healthy and fit man from Thyolo, Malawi, fell from his bicycle. He was seen at Queen Elizabeth Central Hospital (QECH) on the same day and was diagnosed to have a mid shaft transverse fracture of left femur (Fig 2) and an ipsilateral transverse humerus fracture. Both fractures were closed and there were no other injuries. He had stable vital signs on admission and on subsequent measurements. A U-slab was applied to the humerus fracture. The femur fracture was temporarily immobilized with skin traction and pain was managed with oral ibuprofen $400 \mathrm{mg}$ twice daily for 5 days. Conservative treatment was agreed upon and he was kept on traction. He was pain free and not taking any analgesia following the course of ibuprofen mentioned. Twelve days after admission he started to complain of increasing pain and tightness in his left thigh. Sensation and motor function were intact. In spite of regular administration of adequately dosed pethidine, the pain continued and he reported numbness distally the next day. At this time he was sweating and in extreme pain and had tense and tender swelling of the thigh. He was apyrexial. Distal neurovascular examination was normal. Pain was considerably worse while attempting to tighten his quadriceps. An ultrasound scan demonstrated a non pulsatile mass consistent with a haematoma. His hemoglobin dropped to $6.5 \mathrm{~g} / \mathrm{dl}$. He had no history of bleeding diathesis and he denied regular/prolonged use of non steroidal anti inflammatory drugs and had a normal platelet count. His urinalysis was normal.

\section{What is the differential diagnosis of the severe pain in the thigh?}

2. What diagnostic and therapeutic procedures are indicated?

Turn to pg 87 for a discussion of this case.

\section{Wellcome Trust to build an Adult Emergency and Trauma Centre for QECH}

A \$1.6 million Adult Emergency and Trauma Centre (AETC) is to be built at Queen Elizabeth Central Hospital thanks to funds from the Wellcome Trust. According to Professor Robert Heyderman, Director, Malawi Liverpool Wellcome Trust Clinical Research Programme (MLW), the new AETC will help to ease the problem of long waiting periods, reduce overcrowding in the admission wards, act as a teaching facility and provide opportunities for clinical research. He added that the new facility would provide high quality care for patients, offer services like a pharmacy, surgical theatre, laboratory, and short stay ward. The Wellcome Trust has donated $80 \%$ of the budget, the remaining $\$ 325,00$ is hoped to be fundraising locally through MLW. Mr. Tom Chisale, administrator of $\mathrm{QECH}$, has assured that the Ministry of Health is fully committed to this project and will adequately staff the AETC and ensure no stock outs of essential medicines.

The AETC Taskforce, comprising College of Medicine, MLW, and QECH Management is calling on

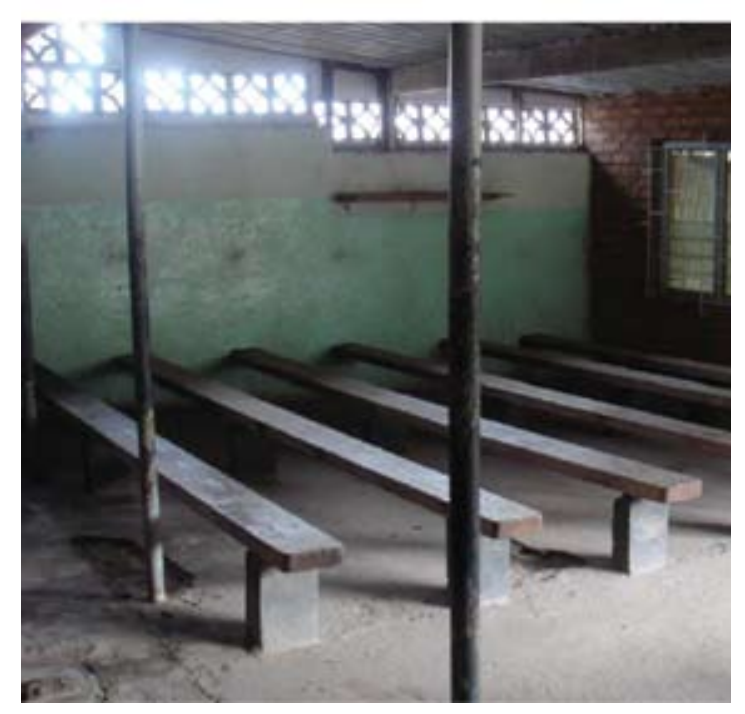

individuals and institutions to kindly donate funds to ensure the completion of this much needed project. Mrs. Tulipoka Soko, Chief Matron, QECH, has said that existing emergency department at the hospital has inadequate space, with only two examination rooms and one treatment room and is also severely under-staffed. Mrs. Soko said initially the casualty department at QECH was planned for only 100 patients but almost 500 patients pass through everyday. "The facility would help to improve contact time with specialists and patients and would help to accommodate the increasing numbers of critically Current waiting area at QECH

New AETC to drastically improve critical care of patients, reduce overcrowding, and shorten waiting time ill patients admitted here." She said. The new unit will have a major and minor surgical theatre, space for sorting out patients based on severity of illness, x-ray facility, laboratory, pharmacy, short stay wards, a teaching room, a large waiting area and offices for doctors, nurses and clinical officers. 


\section{Ward Round - Late presentation of Acute Compartment Syndrome in the thigh}

\section{Continued from page 85}

\section{Discussion}

A clinical diagnosis of acute compartment syndrome of the left thigh was made, with a differential diagnosis of an infected haematoma ${ }^{1,2}$. After cross matching blood the patient was taken to the theatre, and an emergency fasciotomy was performed through a lateral thigh incision as described by Tarlow et al. ${ }^{3}$. The muscle compartment was found to be very tight and a large haematoma (about 750ml) composed of fresh and clotted blood was evacuated. The muscle appeared viable with only mild edema. There was a large cavity in the muscle posteromedially where the distal bone end had impacted, although there was no identifiable source of bleeding. The fracture was reduced and internally fixed with a K-nail (Fig 1\&2) and, since the thigh was now soft, the wound could be closed with minimal tension. Postoperatively he was transfused and closely observed for recurrence of symptoms. Currently he is starting to mobilize with a walking frame and the wound is healing well.

Compartment syndrome is a symptom complex caused by elevated tissue pressure in a closed osseofascial compartment compromising the circulation of muscles and nerves within that compartment $t^{4,5}$. The syndrome may be acute or chronic. In a broad sense, the chronic syndrome follows muscle overuse during exercise while acute compartment syndrome follows trauma such as fractures or contusions. Drugs and venoms can also cause acute compartment syndrome ${ }^{6}$. The commonest sites for compartment syndrome are the in the leg or forearm, and more rarely in the hand, thigh and foot. ${ }^{7}$.This case is unusual in that the symptoms developed 12 days after injury, and after the initial swelling had already subsided. The compartment syndrome was found to be caused by a haematoma which developed presumably after a secondary haemorrhage. The relative lack of stability of the fracture provided by traction may have contributed this. Haematoma formation after thigh contusion has been well described ${ }^{8,}$ 9. Acute compartment syndrome of the thigh caused by haematoma formation after contusion has been described in four reports ${ }^{10-12}$, and late presentation one week after trauma has been observed in two cases ${ }^{13}$. The fibrinolytic activity demonstrated in the haematoma of orthopedic patients 14 could promote continued bleeding and haematoma expansion, and may offer a possible explanation for the delayed development of thigh compartment syndrome our patient.

The case helps to emphasize the need for a high index of suspicion for compartment syndrome in trauma patients who develop severe swelling and worsening pain, and in particular that compartment syndrome can develop secondarily after the initial swelling from the injury has resolved.

\section{Summary}

Acute compartment syndrome of the thigh is rare but has been described as a result of femur fracture and also thigh contusion in sports injury. Emergency fasciotomy has routinely been the recommended treatment. We describe a patient with a closed femur fracture, initially without any complications, who subsequently developed compartment

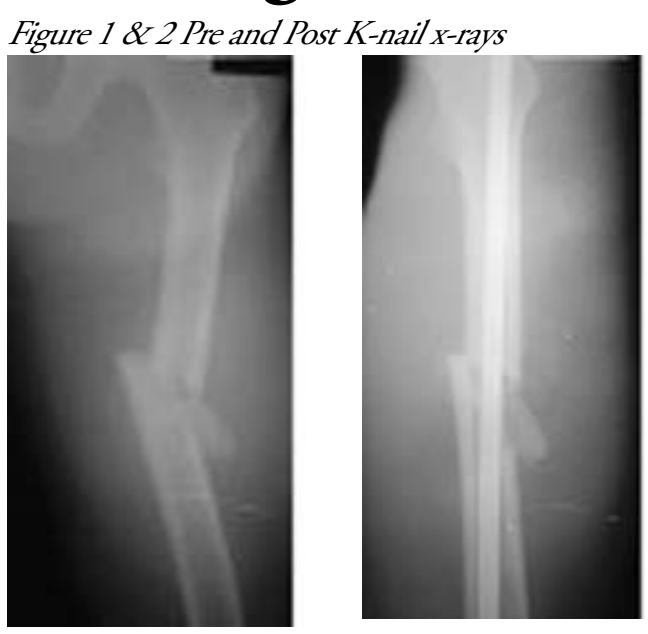

syndrome whilst on traction, and required surgical intervention. He was found to have a large haematoma associated with significant muscle damage in the posterior compartment. This case is unusual in that symptoms started 12 days after injury possibly after manipulation of the leg whilst on traction. Diagnosis is mainly clinical with an earliest alarming sign being disproportional increasing pain on passive stretch of the group of muscles. A high index of suspicion and prompt intervention are required to diagnose and treat compartment syndrome and prevent irreversible damage.

\section{References}

1. Konstantakos EK, Dalstrom DJ, Nelles ME, Laughlin RT, Prayson MJ (December 2007). "Diagnosis and management of extremity compartment syndromes: an orthopaedic perspective". Am Surg 73 (12): 1199-209

2. Ulmer T. The clinical diagnosis of compartment syndrome of the lower leg: are clinical findings predictive of the disorder? J Orthop Trauma. Sep 2002; 16(8):572-7.

3. Tarlow SD, Achtermann CA, Hayhurst J, et al. Acute compartment syndrome in the thigh complicating fracture of the femur. $\mathrm{J}$ Bone Joint Surg Am 1986; 68:1439-1443.

4. Wiley Publishing, Inc.. Webster's New World Medical Dictionary.3rd edition, 2008.

5. Clifford R. Wheeless III Compartment Syndrome Pressure Monitoring

6. N.H. Cawrse, et al. A snake in the clinical grass: late compartment syndrome in a child bitten by an adder JPRAS, Volume 55, Issue 5, Pages 434-435 (July 2002)

7. McQueen MM, Gaston P, Court-Brown CM. Acute compartment syndrome. Who is at risk? J Bone Joint Surg Br. Mar 2000; 82(2):200-3.

8. Jackson DW, Feagin JA. Quadriceps contusion in athletes. J Bone Joint Surg Am 1973; 55:95-105.

9. Rothwell AG. Quadriceps haematoma: a prospective clinical study. Clin Orthop 1982; 171:97-103.

10. Gorman PW, McAndrew MP. Acute anterior compartmental syndrome of the thigh following contusion: a case report and review of the literature. J Orthop Trauma 1987; 1:68-70.

11. Klasson SC, Vander Schilden JL. Acute anterior thigh compartment syndrome complicating quadriceps haematoma. Orthop Rev 1990; 19:421-427.

12. Rooser B. Quadriceps contusion with compartment syndrome: evacuation of haematoma in two cases. Acta Orthop S c a $\mathrm{n} \mathrm{d}$ 1987; 58:170-172.

13. Rooser B, Bengtson S, Hagglund G. Acute compartment syndrome from anterior thigh muscle contusion: a report of eight cases. J Orthop Trauma 1991; 5:57-59.

14. Krohn CD, Reikeras O, Bjornson S, et al. Fibrinogen, fibrin and its degradation products in drained blood after major orthopaedic surgery. Blood Coagul Fibrinolysis 1999; 10:167-71. 